\title{
LA ACCIÓN TERRITORIAL: \\ UNA PROPUESTA CONCEPTUAL Y METODOLÓGICA PARA SU ANÁLISIS
}

\section{TERRITORIAL ACTION:}

A CONCEPTUAL AND METHODOLOGICAL PROPOSAL FOR ITS ANALYSIS

MarCelo Sili

CONICET - Universidad Nacional del Sur, Departamento de Geografía y Turismo, Bahía Blanca, Argentina

\begin{abstract}
R E S U M E N : La acción territorial constituye el insumo básico en la construcción de territorios y del desarrollo territorial. Este concepto permite sustentar el análisis de las formas como la acción pública, la acción colectiva y la acción privada construyen los territorios, muchas veces en forma contradictoria y conflictiva. De esta manera, el concepto de la acción territorial constituye un desafío intelectual, pues permite analizar las realidades territoriales sin caer en el trayectorismo del desarrollo planteado por el paradigma de la modernidad que propone que el destino ineludible de los territorios es el desarrollo (modernización y crecimiento), y abre las puertas a pensar en múltiples y diversos caminos para el futuro de los territorios. Este artículo brinda, finalmente, como producto, ideas y propuestas metodológicas para el análisis de la acción territorial.
\end{abstract}

P A L A B R A S C L A V E S : acción territorial; desarrollo territorial; recursos; actores, metodología.

A B S T R A C T: Territorial action is the basic input for the construction of territories and territorial development. This concept supports the analysis of the ways in which public action, collective action and private action construct territories, often in contradictory and conflicting manners. Thus, the concept of territorial action constitutes an intellectual challenge since it enables us to analyze territorial realities without falling onto the developmental path of the paradigm of modernity, which proposes that the inescapable destiny of territories is development (modernization and growth), and opens the doors to consider various, multiple ways for the future of territories. This article finally provides as a product, ideas and methodological proposals for analysing territorial action

KEYW O R D S : territorial action; territorial development; resources; actors, methodology.

A G R A D E C I M I E N T O S. Este trabajo ha sido realizado gracias al apoyo del CONICET (Argentina), del Fondo para la Investigación Cientifica y Tecnológica de la Agencia Nacional de Promoción Cientifica y Tecnológica (Argentina) y del Centro "Investigación para el Desarrollo" (Paraguay).

DOI: https://doi.org/10.22296/2317-1529.2018v20n1p11 


\section{INTRODUCCIÓN}

América Latina ha vivido un profundo proceso de transformación territorial en las últimas décadas. Así, de un modelo territorial estructurado por grandes ciudades y áreas rurales donde cohabitaban grandes latifundios con la agricultura familiar, con una diversidad de pueblos y pequeñas ciudades ligadas a la prestación de servicios rurales, se pasa a otro modelo de organización territorial más complejo, caracterizado por el creciente peso de las ciudades de todos los tamaños (incluyendo ciudades medianas) (VEIGA, 2002), con nuevas dinámicas de relación urbano rural (TACCOLI, 2006), con la emergencia de nuevas actividades productivas más diversificadas (BERDEGUÉ et al., 2012). Pero siempre sobre la base de la explotación de los recursos naturales y la utilización de nuevas tecnologías y modelos de producción, que continúan privilegiando la deslocalización de la renta y la polarización social, y con dinámicas demográficas más complejas, donde si bien se mantiene el éxodo rural, también se observan efectos de contraurbanización (SILI, 2016). Este nuevo modelo de organización territorial no está exento de crecientes tensiones y problemas sociales y ambientales y de fragmentación social y económica (COMISIÓN ECONÓMICA PARA AMÉRICA LATINA, 2016).

Detrás de este escenario de transformación territorial, sobre el cual hay, además, múltiples hipótesis e investigaciones analíticas, es posible observar la emergencia de numerosas iniciativas como planes, programas y proyectos de desarrollo de todo tipo, con el objetivo de superar los procesos de crisis de los territorios que se mantienen marginales a las dinámicas de crecimiento económico, o captar nuevas oportunidades en los territorios más dinámicos, intentando así consolidar escenarios de mayor desarrollo y calidad de vida. Esta multiplicidad de iniciativas se construyen desde los Municipios locales, provinciales y nacionales, pero también desde diferentes sectores sociales y productivos, configurándose un ambiente político institucional sumamente complejo, de una gran densidad de iniciativas, muchas veces signadas por la superposición, los conflictos y la competencia entre ellas, lo cual, en la mayor parte de las veces, lleva a la pérdida de eficacia y de impacto de las mismas (RIFFO, 2013). Un elemento distintivo de este ciclo histórico signado por la globalización es la "sobreabundancia” y la falta de coordinación de iniciativas y proyectos, el concepto de desarrollo aparece con múltiples significados y representaciones por parte de los actores locales y regionales, lo cual configura una situación muy compleja e incierta, pues no existe una orientación clara de hacia dónde se deben dirigir las múltiples iniciativas en marcha, y qué tipo de escenario deseado de desarrollo se pretende construir.

Teniendo en cuenta esta situación, el interés de este trabajo radica en definir un conjunto de ideas, conceptos y metodologías, para analizar y comprender cómo los diversos actores, organizados a través de la acción pública, la acción colectiva o la acción privada, construyen, a partir de su vida cotidiana, estas acciones y procesos de diferente índole, institucionalizados a veces en planes o proyectos de desarrollo o no, en vistas a alcanzar escenarios del desarrollo para sus territorios, acciones y procesos que circunscribimos bajo el concepto de acción territorial.

Se entiende a la acción territorial como un proceso multiactoral de construcción y organización de territorios, pero cuyo devenir es incierto, pues depende de numerosas variables y condiciones y, sobre todo, del ideario de desarrollo y del poder que los propios actores involucrados posean y movilicen (POUTHIER, 2013). Los resultados 
de la acción territorial pueden ser dispares, ya que no siempre son escenarios deseados de desarrollo. Al contrario, en muchas ocasiones la dinámica de la acción de los múltiples actores genera situaciones de mayor desequilibrio, deterioro o empobrecimiento de los territorios. En este sentido, la noción de acción territorial nos permite pensar el futuro de los territorios de una manera más descarnada y real, sin caer en la trampa del trayectorismo occidental (APPADURAI, 2015) por la cual los territorios están sujetos y condicionados a seguir una línea de tiempo que inevitablemente nos conduce a escenarios de superación y progreso, y que siempre implicó que toda política e intervención en los territorios debe servir necesariamente para remover los obstáculos que frenan o impiden avanzar hacia ese escenario predestinado de progreso.

Para poder definir y tornar operativas dichas ideas y posibles metodologías para el análisis de la acción territorial, primero será necesario partir de una breve conceptualización del territorio, de su trayectoria temporal y por sobre todo del concepto de desarrollo territorial, concepto llave en este contexto histórico en América Latina, siempre anunciado, pero sobre el cual existen fuertes divergencias en torno a su significado y su sentido.

\section{UNA BREVE REFLEXIÓN SOBRE EL TERRITORIO Y EL DESARROLLO TERRITORIAL}

Los profundos cambios tecnológicos y la intensificación de las interdependencias entre países y territorios (SASSEN, 1991; CASTELLS, 1999; DICKEN, 2010) no solo han transformado la naturaleza de la relación espacio-sociedad y también la conceptualización que se hace de la misma. Los debates sobre la relación globalización - espacio han oscilado entre enfoques que plantean la tendencia hacia la disolución del espacio como fricción o barrera a los procesos económicos, sociales, culturales y políticos y, por tanto, la emergencia de un mundo que tiende hacia la homogeneidad (OHMAE, 1990), y por otra parte, enfoques que postulan exactamente lo contrario, esto es, la creciente relevancia de las diferencias y las especificidades locales, la afirmación de identidades, los conocimientos y aprendizajes locales, etc. (MASSEY, 1991; VELTZ, 1992; PORTER, 2000). Esta segunda posición ha dado lugar a una profusa reflexión sobre la relación espacio sociedad, que visualiza al espacio como una dimensión de la sociedad, y no como un contenedor de la sociedad o como un reflejo de los hechos sociales y económicos. Bajo esta línea argumental, el espacio tiene un rol activo y estructurante en la construcción de la sociedad, y a su vez el espacio es estructurado por lo social (GIDDENS, 1984; MASSEY, 1985). Este avance conceptual que implica la redefinición de la relación espacio-sociedad en las ciencias sociales influye con fuerza en los nuevos enfoques del desarrollo regional y local que emergen en la década de los 80 s, que se expresarán principalmente en la perspectiva del desarrollo endógeno (MONCAYO JIMÉNEZ, 2001).

Esta redefinición de la relación espacio-sociedad nos lleva a posicionar al territorio como pieza clave de las políticas de desarrollo. La base etimológica de territorio hay que buscarla en el vocablo latin territorium que, derivado de terra, significa porción de tierra apropiada. Por lo tanto, territorio es la porción de tierra que puede ser utilizada, organizada y controlada en función del deseo de una persona o un grupo de personas. Esta acepción, vinculada al sentido del poder y el control de un espacio por parte de 
una persona, fue evolucionando paulatinamente, entendido para algunos como un "espacio apropiado por un grupo social con conciencia de su pertenencia y apropiación” (BUSTOS CARA, 2008, p. 16). Otra definición cercana a ella plantea que el territorio es un espacio geográfico con un sentido, un sentido individual y colectivo sobre su realidad actual pero también con un significado u orientación hacia el futuro.

De esta manera entendemos al territorio como un espacio geográfico que carga con un conjunto de intencionalidades políticas, sociales, productivas, culturales (proyectos) que se plasman o concretan en función de las capacidades de los actores o los grupos de actores para llevarlas a cabo (Capacidad de acción). Así, el territorio no es entonces un simple soporte físico, sino la "arena" donde los grupos sociales construyen un devenir. Por lo tanto, las características de estos territorios no van a depender sólo de sus condiciones naturales, ni de su configuración espacial, sino también de las formas en cómo se articulan entre sí los proyectos de cada uno de los actores y cómo a partir de esta articulación son capaces de valorizar y gestionar el lugar y construir un proyecto de futuro.

La idea de un proyecto de futuro nos remite al hecho que todo territorio tiene una dimensión histórica, una historia a través de la cual se define su trayectoria temporal. Dicho proyecto de futuro no es el simple producto mecánico de la agregación de proyectos individuales, sino el producto de complejas relaciones, conflictos y representaciones sociales. "La objetividad nos obliga a reconocer el carácter teleonómico de los seres vivos (y de los territorios), a admitir que en sus estructuras y performances ellos realizan y persiguen un proyecto" (MONOD, 1970 apud LE MOIGNE, 1989, p. 124), construcción que se hace condicionado por las características del medio físico y del contexto global del territorio. Es a través de este proyecto de futuro y de las acciones e iniciativas que los hombres ponen en marcha para construir el mismo que se puede interpretar un territorio, como lo afirma Le Moigne (1989, p. 136) "es en relación al proyecto del sistema (el territorio) y no con respecto a la estabilidad observable que la acción pueden ser interpretadas. El objeto (territorio) define su ideal de estabilidad, no por la no variabilidad de su estructura, sino por la satisfacción de sus proyectos". En otras palabras, en su evolución histórica, el sistema (territorio) va definiendo una trayectoria evolutiva en tanto realiza el proyecto teleonómico que se fijó o diseñó socialmente, tanto de manera explícita o implícita. La performance de dicho territorio va a medirse entonces como la capacidad que tiene el mismo para mantenerse, renovarse y lograr concretar los proyectos planteados a través de una compleja y densa red de acciones de sus actores. Un desafío mayor en términos de política territorial, expresada y formalizada a través de una estrategia de desarrollo territorial, es definir con claridad el escenario hacia dónde avanzar y a partir de allí definir los instrumentos y los mecanismos para construir dicho escenario. No obstante, es necesario reiterar aquí la necesidad de salir de la trampa del trayectorismo, es decir, del relato social de la inevitabilidad de caminar hacia escenarios de progreso y modernidad, y pensar el futuro de los territorios como una construcción social y cultural pensada desde la propia realidad y las representaciones y necesidades de los actores del mismo, y claramente desenmascarada la idea de una trayectoria inevitable hacia el progreso. Esto no impide pensar que el progreso y la modernidad pueda ser considerado como una vía a seguir, pero claramente esta vía debe ser discutida y consensuada.

Visto de esta manera, la definición del proyecto de futuro del territorio (VANIER, 2015) es clave para la estructuración de la acción y de políticas, pues favorece la 
convergencia y concertación de múltiples actores en torno a un ideario del territorio (APPADURAI, 2015). Así, los objetivos y escenarios deseados de futuro permiten construir utopías capaces de movilizar y concertar diferentes actores de la sociedad, favoreciendo la constitución de un proyecto territorial a partir del cual la solidaridad y el juego de competencias se ordenan. De esta manera la definición de un proyecto de futuro con sus objetivos actúa como marco de regulación de las energías y los conflictos sociales, en función de un proyecto colectivo.

Los objetivos de desarrollo aparecen, entonces, como el faro que orienta y hacia el cual se dirigen las acciones de una sociedad. Actúan como utopía movilizadora de la sociedad, pues lo que no es todavía actúa sobre la realidad actual. Lo importante es construir esta utopía de futuro que claramente podría ser diferente a la trayectoria impuesta por la lógica de la modernización, enraizada en el capitalismo y el modelo de acumulación y crecimiento Así, el territorio se define y gestiona desde el presente a partir de un proyecto de futuro, poniendo en valor el legado histórico para adecuarlo a las exigencias actuales (FRANÇOIS; HIRCZAK; SENIL, 2006; LAJARGE, 2011). Interesa la tensión que se produce entre el presente y los objetivos y escenarios deseados para el futuro, pues esta tensión es la que promueve la acción territorial, materia prima de cualquier proceso de desarrollo territorial.

Desde otro punto de vista, es importante considerar que para poder construir dicho escenario y proyecto de futuro, los objetivos deben estar claramente explicitados y ser capaces de ser leídos y comprendidos por la sociedad en su conjunto, por lo tanto deben ser parte del universo de referencia de los actores. La evidencia en América Latina en las últimas décadas muestra que muchos de los fracasos de las políticas de desarrollo se producen debido a:

1. Por un lado, el divorcio que se plantea en el universo cultural de los diferentes sectores sociales de los países, entre las políticas de modernización y desarrollo planteadas por las élites, y el universo de referencia cultural del resto de la sociedad (RIST, 2013). En este caso se observa como muchos países, regiones y localidades han caído en la trampa del trayectorismo.

2. Por la carencia de una definición clara de un proyecto como guía hacia dónde encauzar las acciones de los múltiples y diversos actores, proyecto que deberá ser construido sin caer en la trampa del trayectorismo. La experiencia muestra que los "territorios huérfanos de futuro", es decir, aquellos que no pueden definir una imagen o escenario de futuro o una imagen realista, coherente con las condiciones estructurales locales, difícilmente puedan alcanzar escenarios de mayor desarrollo y sólo alcancen la mejora de ciertas variables del territorio (por ejemplo crecimiento productivo). Esto constituye un desafío mayor, pues es necesario construir un proyecto de futuro que sea capaz de sintetizar las múltiples voces y no sólo aquellas que tienen más poder o capacidad para imponer sus propios proyectos.

Ahora bien, dentro de la lógica de la globalización y del paradigma de la modernización y el crecimiento imperante en las últimas décadas, los proyectos de futuro de los territorios en América Latina han estado claramente dominados por dos grandes elementos. En primer lugar, por la necesidad de valorizar los recursos (especialmente recursos naturales), generar nuevas innovaciones y mejorar las condiciones de producción, con el objetivo final de lograr una mayor competitividad del territorio en términos económicos productivos, lo cual conduciría según Schejtman y Berdegué 
(2004) a una mejora de la calidad de vida en la región. En segundo lugar, por la necesidad de modernizar los modelos de gestión y gobernanza, pues se asume que en este nuevo contexto global, donde prima una nueva lógica de redes, los territorios deben reinventar sus mecanismos de gestión, de manera que se puedan resolver, administrar y reorientar las profundas transformaciones políticas y económicas y garantizar buenas condiciones sociopolíticas para asegurar el desarrollo productivo. Dentro de este contexto, las consideraciones sobre la identidad, el patrimonio, las infraestructuras, los equilibrios socioespaciales y la planificación territorial entre otras, si bien han estado sumamente presentes, las mismas han sido subsidiarias y han estado supeditadas a estos dos grandes objetivos de competitividad territorial y gobernanza.

Más allá de l'air du temps y del paradigma dominante, es claro que un proyecto de futuro asociado al desarrollo territorial no se agota en la competitividad y la gobernanza, sino que se deben considerar múltiples dimensiones de carácter eminentemente territoriales, pues lo que constituye el objeto y sujeto de cualquier proceso de desarrollo es el territorio en sí mismo, y no un sector productivo o un grupo de actores solamente (SILI, 2010). Teniendo en cuenta ello, desde un punto de vista prescriptivo, y como solo hecho para localizar el referencial de pensamiento que nos moviliza en términos de ideario de desarrollo, definimos al desarrollo territorial (CAMPAGNE; PECQUEUR, 2014) como un escenario deseado de futuro que se caracterizaría por las siguientes imágenes:

1. La existencia de una real capacidad de gestión y control del territorio por parte de los actores concernidos por dicho territorio.

2. Una identidad territorial fortalecida, fuente de innovación y desarrollo cultural que le permite a la sociedad comprender su existencia, su lugar y su relación con el mundo.

3. Un hábitat, un paisaje y un ambiente sano, garante de una elevada calidad de vida segura y de sostenibilidad del empleo.

4. Una organización espacial que favorece la integración social y económica y minimiza los conflictos por el uso del suelo y los recursos.

5. La presencia de infraestructuras y equipamientos adaptados a las condiciones del territorio y con capacidad para mejorar la calidad de vida, mitigar los riesgos y promover el empleo de calidad.

6. La existencia de un ambiente de innovación y valorización de la multiplicidad de los recursos del territorio de manera de sostener sistemas de producción con empleo de calidad.

\section{LA ACCIÓN TERRITORIAL, COMO MECANISMO DE CONSTRUCCIÓN DEL TERRITORIO Y EL DESARROLLO TERRITORIAL}

En función de esta concepción del territorio y del desarrollo territorial, la acción territorial puede ser definida como el conjunto de iniciativas y acciones que ponen en marcha los diferentes actores involucrados en el territorio, capaz de dotar de sentido, significado y orientación al mismo, generando funcionalidades y legitimando localizaciones de infraestructuras, sistemas productivos u órdenes normativos que se despliegan en un espacio, calificándolo en función de su propio sentido del presente y 
del futuro, y en función del ideario de desarrollo que poseen los actores comprometidos con el mismo (BUSTOS CARA, 2009). En otras palabras, la acción territorial es el proceso a partir de la cual una sociedad construye su proyecto territorial asociado a su propio ideario de desarrollo.

La acción territorial es necesariamente un proceso complejo que integra el pasado, dada la memoria y las estructuras físicas heredadas, el presente, por las condiciones de contexto actuales, y el futuro de acuerdo a las imágenes y representaciones que tenemos sobre ese futuro deseado y que determinan las intencionalidades en la toma de decisiones. Así, la acción territorial integra y coordina necesariamente múltiples y diversas iniciativas de actores con temporalidades y ritmos de vida muy diferentes, pues todos los actores y los grupos sociales involucrados no necesariamente siguen las mismas lógicas y las mismas rítmicas culturales. De allí que la acción territorial es también un claro generador de conflictos entre actores y grupos sociales, pues las acciones de unos chocan o limitan las acciones de otros actores. Así, las acciones territoriales no siempre son horizontales, ni benéficas para todos los actores o habitantes de un territorio, al contrario, pueden tratarse de acciones que contradicen las ideas y representaciones de desarrollo de otros actores. Las estrategias para superar los conflictos que se generan a partir de la acción territorial de múltiples actores es una tarea sobre la cual no existen recetas claras, sino que constituye una tarea política y técnica de envergadura. De esta manera, la acción territorial debe ser entendida desde una perspectiva de construcción y transformación de la sociedad y el territorio, como un ir haciendo en el territorio, contenidos por una estructura que condiciona (medio físico, condiciones político institucionales, etc.), pero también con un margen de maniobra y autonomía por parte de los sujetos (BUSTOS CARA, 2009), lo cual permite pensar y promover una visión optimista sobre las posibilidades de intervención y construcción de escenarios deseados. La acción territorial se encarna, entonces, en un sujeto intencional, orientado hacia el futuro, un sujeto competente, pues tiene capacidad para movilizar recursos, conocimientos e información sobre la realidad. Esto supone un reconocimiento de que "el actor no existe fuera de un sistema que define su libertad y la racionalidad que puede utilizar en sus acciones, pero también se reconoce que el (sistema) territorio no existe sin el actor que le da vida y puede transformarlo" (CROZIER; FRIEDBERG, 1977, p. 11).

En síntesis, la acción territorial constituye el conjunto de iniciativas que los actores ponen en marcha para construir y dar sentido a un territorio, estas acciones son situadas en un espacio determinado, un espacio de interacción (territorio de acción) incluido dentro de un contexto estructurante más amplio (territorios y sociedades que los abarcan y contienen) y están estructuradas bajo un modelo de gobernanza, condicionadas por las normas o reglas formales e informales vigentes y el intercambio de recursos, que se caracterizan por ser escasos y estar inequitativamente distribuidos (MANZANAL; PONCE, 2012).

\section{¿QUÉ TIPOS DE ACCIÓN TERRITORIAL?}

La acción territorial son todas aquellas iniciativas capaces de organizar y transformar un territorio, y que pueden ser planificadas, pautadas y organizadas por una sociedad o por diferentes grupos sociales (la gestión del agua por parte de un grupo de productores, la organización de una feria para la valorización del patrimonio cultural 
local, etc.) o por individuos (la creación de una fábrica, la realización de cultivos, etc.), pero también incluye todas las acciones cotidianas que "no surgen necesariamente de un plan consciente, sino simplemente de lo que la gente hace, siente, percibe y llega a articular en su búsqueda de significado para su vida cotidiana. Tales prácticas crean espacios heterotópicos en todas partes" (HARVEY, 2013, p. 15). En el mismo sentido Gumuchian y otros plantean "el territorio debe ser pensado a través de un conjunto de microeventos, entendiendo por tales las actitudes y los comportamientos de los actores en el territorio" (GUMUCHIAN et al., 2003, p. 48). Muchos de estos microeventos, o acciones individuales o colectivas, producen resultados cuya dimensión territorial es menor. Sin embargo, la red de estas acciones se transforma en un momento dado o confluye en la construcción de un proyecto territorial. Harvey señala al respecto "No tenemos que esperar a que la gran revolución constituya esos espacios [...]. Lo espontáneo confluye en un momento de irrupción cuando diversos grupos heterotópicos ven de repente, aunque solo sea por un momento efímero, las posibilidades de la acción colectiva para crear algo radicalmente diferente" (HARVEY, 2013 , p. 15). Es decir, un proyecto territorial que tiene un sistema de acción que le es propio. Un ejemplo claro de esto se da cuando un conjunto de asociaciones de productores agropecuarios se organiza para crear una feria local, la cual luego da lugar a la creación de una marca territorial y posiblemente a la puesta en marcha de un proyecto más integral de desarrollo rural, con inversiones en infraestructuras y otras acciones de planificación del uso del suelo por ejemplo. Este encadenamiento de acciones de menor a mayor complejidad termina por contribuir a la definición de un proyecto territorial con una clara definición de un escenario de futuro.

Desde el punto de vista de su complejidad, hay acciones territoriales que pueden ser consideradas como iniciativas cotidianas de los actores en términos individuales (MOINE; FAIVRE, 2013, p. 3), pero también hay acciones territoriales de mayor complejidad y envergadura, llevadas a cabo por uno o múltiples actores y con más fuerza y capacidad para organizar y estructurar el territorio. Así, la creación de una ruta, la ocupación de tierras por parte de grupos campesinos, la instalación de una industria o un supermercado en un área residencial, la planificación de un espacio urbano, la valorización de un recurso patrimonial local, etc., son acciones de mayor complejidad y envergadura de mayor visibilidad y estructuración territorial.

Las acciones pueden también ser de distinta naturaleza o finalidad. En muchos casos las acciones pueden ser generales, es decir, se interviene en forma integral, por ejemplo, a través de la puesta en marcha de un plan de desarrollo del territorio, lo cual no quiere decir que esta acción sea eficaz, incluyente y participativa, pues puede ser (como de hecho suele ser en numerosas oportunidades) una acción vertical sin compromiso ni participación de los actores locales. En otros casos las iniciativas de los actores se circunscribe a temas específicos, acciones territoriales de carácter social, con el objeto de mejorar la calidad de vida de la población, por ejemplo, la organización de nuevas actividades educativas en el territorio, un proyecto de valorización cultural y patrimonial, la implementación de un plan de salud, la creación de una sala médica, etc.; otras pueden ser de desarrollo económico, con el objetivo de generar mayor actividad y mayores ingresos, como por ejemplo la creación de empresas, la organización de un grupo de productores en torno a un problema de comercialización y agregado de valor de su producción, la creación de un parque industrial, la introducción de innovaciones productivas, etc.; pueden tratarse de creación de infraestructura, cuan- 
do implique la construcción y mejora de los elementos o servicios necesarios para el funcionamiento en sociedad; o de ordenamiento territorial, cuando la dinámica y la acción de los actores se circunscriba a la organización del uso y ocupación del espacio (JIMÉNEZ, 2016). Estas acciones con finalidades específicas pueden estar bien coordinadas y ser complementarias con otras acciones, o bien puede suceder, tal como lo muestra la experiencia, que sean acciones que no se articulan ni complementan, o aún más, se contradigan entre sí.

Una última característica del tipo de acción territorial lo constituye su nivel de estructuración, institucionalización o formalización a través de normas e instituciones. Así se pueden identificar una acción territorial formalizada cuando las mismas se legitiman mediante normas u organismos específicos que surgen para regular su desarrollo. Así, por ejemplo, la construcción de un plan de viviendas por parte del estado está estructurada por reglas burocráticas y administrativas bien claras por parte del sector público, o la puesta en marcha de un plan de regulación urbana, etc. Hay también acciones territoriales que son de cierta envergadura y que se encuentran en procesos de estructurarse o formalizarse institucionalmente, sin que muchas veces este proceso sea completo. Y por último, hay innumerables acciones que no tienen ningún tipo de estructuración o institucionalización, presentando actuaciones más bien esporádicas de acuerdo a la intencionalidad de los participantes y a las necesidades que se presenten en el territorio.

\section{¿DÓNDE SE PRODUCE LA ACCIÓN TERRITORIAL?}

Cualquier acción territorial se produce dentro de un espacio de interacción en donde se construye un vínculo social y se intercambian recursos (KNOEPFEL et al., 2007; SUBIRATS et al., 2008). Estos espacios pueden tener diferentes dimensiones, pero son, ante todo, los espacios de acción cotidiana de los actores. Esto puede ser un paraje rural, un pueblo y su área de influencia, una pequeña microrregión, una provincia, etc. Lo que define el territorio de acción no son las fronteras o los límites políticos administrativos, sino el poder de actuación o la legitimidad que los actores tienen sobre un territorio. Así por ejemplo, un productor agropecuario tiene un ámbito de acción centrado en su explotación, pero también en su paraje rural y en su pueblo o pequeńa ciudad en tanto interviene en redes de organización locales promotoras de actividades vinculadas a la vida cotidiana de ese lugar. Pero también hay actores cuyos ámbitos de acción son de otros niveles escalares, por ejemplo un gobierno provincial tiene un ámbito de actuación de toda la Provincia. Una organización empresarial e industrial que lleva adelante acciones de promoción y desarrollo de la actividad industrial de su sector tiene un ámbito de acción que sobrepasa lo local y que se despliega en el territorio de intervención que puede ser local o provincial o nacional. Estos espacios se encuentran dentro de un contexto espacial estructurante o ámbito de referencia (territorio nacional o provincial) que es el que define ciertas condiciones a las cuales se sujetan los actores.

Sin embargo, lo que es clave es que si bien hay acciones que tienen un claro registro o inscripción local, o provincial o nacional, la acción territorial es un proceso multiescalar, es decir, una construcción que se realiza con estrechos vínculos entre los múltiples niveles de organización del territorio. Así, las acciones que dan sentido y direccionalidad a un territorio pueden surgir mayormente de una fuerza endógena, 
que emerge del propio territorio local a partir de sus capacidades, pero también puede estar impulsada desde una fuerza exógena preponderante, sea esta provincial, nacional o internacional o puede estar controlada o regulada por fuerzas o condicionantes que operan en otros niveles escalares. Esta visión de proceso multiescalar debe permitir superar interpretaciones muy localistas y concentradas exclusivamente en las fuerzas o factores endógenos. La experiencia muestra al respecto que aquellos lugares que se pueden conectar con actores y empresas de otros niveles escalares, pueden generar vínculos portadores de innovación y de nuevas oportunidades de acción. En otras palabras, las posibilidades de construir acciones virtuosas en un territorio va a depender en muchas ocasiones de la capacidad de los actores de articular esos múltiples niveles escalares en torno al proyecto del territorio.

Desde otro punto de vista, la experiencia muestra que para que una acción territorial pueda ser eficaz y contribuir a la generación de circuitos virtuosos de desarrollo territorial debe ser construida al amparo de marcos regulatorios estables y previsibles que le dé certeza a los actores involucrados, es decir, se debe contar con políticas globales estructuradas por los niveles escalares superiores, de manera que actúen como "paraguas" de protección o marcos de referencia para la acción pública, privada y colectiva de nivel local. La acción territorial construida sin marcos de referencias claros suelen profundizar los procesos de fragmentación de los territorios. Así, por ejemplo, si a nivel local un intendente y un grupo de vecinos ponen en marcha una iniciativa ambiental que no tiene ningún correlato o marco legal que lo contenga a nivel provincial, es muy probable que estas iniciativas fracasen o tengan dificultades de salir adelante. Las experiencias ambientales son muchas veces loables a nivel local, pero pueden no ser exitosas dado que no existen políticas o normativas de orden general que los contenga. Otro caso muy frecuente es cuando un municipio o gobierno local pretende poner en marcha una estrategia de desarrollo con un sentido de futuro determinado. En muchos casos, dichos escenarios de futuro no tienen su correlato a nivel regional o nacional donde los modelos de futuro planteados no son muchas veces similares o, aún más, pueden ser completamente contradictorios con el modelo local.

\section{¿QUIÉN PRODUCE LA ACCIÓN TERRITORIAL?}

Los actores concernidos en la construcción de la acción territorial son múltiples. En función a su forma de organización, podemos clasificar a los actores en públicos, privados y colectivos. La acción pública es ejercida por aquellos sujetos que basan su propósito de intervención en el hecho de que representan a los ciudadanos, y se rigen por la estructura política administrativa del Estado. La lógica de acción implica actuar en función de aquellas alternativas que mejoren la capacidad para obtener consenso político, promoviendo los acuerdos y compromisos que sean necesarios. Real-Dato (2009) señala que los actores públicos están integrados por: el gobierno, que representa el poder ejecutivo en los diferentes niveles de competencia; la burocracia y la administración pública, que ejecuta las decisiones gubernamentales; y el parlamento y los órganos de justicia, que constituyen el poder legislativo y judicial. Dente y Subirats (2014) en tanto, identifican: a los actores políticos, que participan del proceso decisional en términos generales o con referencia explícita a la cuestión que se esté tratando; y a los actores burócratas, que basan su intervención en la consideración que las reglas legales les atribuyen una responsabilidad determinada en el procedimiento. 
Los actores privados, en tanto, reúnen a los individuos, empresas y corporaciones que intervienen en el territorio a partir de un interés específico que implica en muchas ocasiones la maximización de beneficios económicos o el logro de beneficios sociales o culturales. La dinámica que se haga preponderante en el territorio incidirá directamente sobre sus intereses, sea por los costes que implica afrontarla o por la posibilidad de incrementar alguna de sus posiciones en el espacio social (BOURDIEU, 2005).

Finalmente la acción colectiva, implica la integración de distintos actores en forma de asociación para asumir la representación de intereses que no pueden actuar por ellos mismos, tales como la salud, la educación, la igualdad, la protección del medio ambiente, la seguridad. Estos intereses generales suelen estar lejos de encontrarse entre los prioritarios de los actores políticos y privados, por lo que pujan por sus propios mecanismos de participación (CROZIER; FRIEDBERG, 1977). El propósito de intervención busca contribuir al bien común, incorporando ciertas estructuras organizativas que asumen la tutela de intereses generales y los representan en las distintas instancias que se requiera para evitar que sean desatendidos (BUSTOS CARA, 2008; DENTE; SUBIRATS, 2014).

Esta clasificación de los actores en públicos, privados y colectivos de acuerdo a la lógica de intervención asocia a los sujetos sociales a determinados comportamientos y al uso de recursos específicos. Si bien estos actores se reúnen en grupos relativamente homogéneos, se encuentran atravesados por la pluralidad que circunscribe cualquier comunidad, en muchos casos conformando sub-grupos específicos, que compiten por los mismos recursos en juego. Esta fragmentación interna influenciará también en las estrategias finalmente implementadas, dando cuenta de vínculos de competencia tanto externos como internos.

El abordaje empírico del tipo y organización de los actores requiere identificar varios elementos.

- En primer lugar, el tipo y la densidad de los actores involucrados en la acción

- En segundo lugar, las lógicas, representaciones e imaginarios que ellos movilizan y que fundan y legitiman su acción, pues son estas lógicas y representaciones las que en definitiva guían las acciones de los hombres. Identificar estas representaciones e imaginarios permite reconocer los intereses y motivaciones que existen en torno a un determinado territorio.

- En tercer lugar, las funciones que cada actor desempeña en el entramado socio-territorial, el tiempo que llevan participando de esa gestión y los principales escenarios formales o informales en los que se vinculan.

El abordaje pormenorizado de estas cuestiones permitirá identificar las razones por las que se han producido determinadas dinámicas, comprendiendo las modificaciones paulatinas pero sostenidas que accionan el territorio. Así, tal como lo argumenta Abramovay (2006, p. 67):

El estudio de los territorios desde el ángulo de las fuerzas sociales que los componen no es sólo una invitación a análisis empíricos bien fundamentados sobre su constitución - más que de recomendaciones de política - sino que allana el camino hacia la comprensión de los cambios que nuevas fuerzas sociales pueden imprimir a la manera como están hoy organizados.

Reconocer a los territorios como campos de disputa, en los que se ponen frente a frente los protagonistas, permite explicar las distintas transformaciones que se cristalizan en el espacio desde sus propias bases. 


\section{LOS RECURSOS EN JUEGO EN LA ACCIÓN TERRITORIAL}

Otra de las variables claves constitutivas de la acción territorial, la constituyen los recursos a partir de los cuales los actores intentan imponer sus valores e intereses en la estrategia implementada. Subirats et al. (2008, p. 94) definen recurso como "un activo de materias primas de las que los actores públicos y privados se sirven para llevar a cabo sus acciones".

Es importante tener en cuenta que no existen recursos de los territorios "en sî", sino que estos surgen de la intencionalidad, la movilización y la cooperación de los actores que con cierto grado de autonomía los construyen a partir de su relación con el sistema de producción (KEBIR, 2006; LANDEL; PECQUEUR, 2011). Así, cada sujeto territorial estructurado por diferentes elementos de la economía, la cultura y la naturaleza, interactúa con los bienes que le vienen dados (fauna, flora, patrimonio, conocimiento, historia, etc.), imbuyéndolos de un mayor o menor valor en función de sus objetivos e intereses en el entramado territorial, seleccionando aquellos objetos que considera más relevantes para su estrategia y excluyendo a otros. Es cada sociedad y sus actores, la que activa sus componentes y los resignifica en el tiempo en función a nuevos contextos, nuevos usos y nuevas demandas.

Los recursos territoriales son escasos y se distribuyen desigualmente entre los miembros de una sociedad. Los actores que ejercen dominio sobre alguno de los activos en juego son quienes finalmente logran influir en la entramado decisional, intercambiando los diferentes recursos de los que disponen o que pueden movilizar, mientras que las mayorías que no controlan los recursos suelen acompañar o bien rechazar pasivamente estos procesos.

Para el abordaje empírico de estas dinámicas, Knoepfel et al. (2007) y Subirats et al. (2008) aportan una tipología de recursos fácilmente identificables:

- Recursos patrimoniales o infraestructura: son el conjunto de bienes tangibles que disponen los actores, pueden ser elementos naturales y culturales, la infraestructura, el equipamiento, las instalaciones, etc.

- Recurso jurídico o derecho: son las bases legales y reglamentarias de derecho constitucional, civil y administrativo, que organiza el contenido y la selección de los otros recursos. Es administrado principalmente por los actores públicos, rigiendo la organización del territorio.

- Recursos humanos o personales: refieren a la formación de los participantes en el entramado territorial. La complejidad creciente de los problemas colectivos, como sucede con el desarrollo territorial, atribuye un rol cada vez más importante a este recurso, en términos cuantitativos y cualitativos.

- Recursos económicos: aluden a los medios financieros necesarios para implementar las acciones.

- Recursos cognitivos o información: son los conocimientos vinculados a resolver los problemas, los avances científico-tecnológicos como otros saberes no formales vinculados a la vida en sociedad.

- Recursos relacionales u organización: es la capacidad de organizar la vinculación entre actores y los valores colectivos de una comunidad (Hernández, 2016).

- Recursos de confianza o consenso: hace referencia a la conformidad y acuerdo entre los actores respecto a las acciones y objetivos a emprender, lo cual brinda mayor o menor legitimidad a las acciones. 
- Recursos cronológicos: refieren al tiempo necesario para que la acción sea implementada.

- Recursos de apoyo político: constituyen la legitimidad primaria necesaria para el impulso o modificación de una acción. Se obtiene a través de diferentes mecanismos, especialmente el voto.

- Recurso de fuerza: refiere a la coacción legítima mediante la violencia física para obligar a los actores a modificar determinada conducta o para expresar su desacuerdo, en forma comúnmente de amenaza, intimidación, represión y encarcelamiento.

La dotación de estos activos entre los diferentes actores, así como su producción, gestión, explotación e intercambio va a influir directamente en la acción territorial. Todo análisis de la acción territorial debe contrastar empíricamente qué recursos se utilizan, combinan o sustituyen, el carácter público, privado o colectivo y la evolución de estos activos en el tiempo.

Este análisis de los activos en juego en el territorio permitirá describir los principales recursos invertidos en las acciones, la estrategia impulsada y bajo el dominio de qué agentes se encuentran, contribuyendo a identificar los propósitos explícitos e implícitos de la dinámica territorial, los actores beneficiados y afectados con la iniciativa. La facilidad o dificultad de emprender procesos de activación territorial dependerá de la distribución de los recursos entre los participantes con lógicas de acción y objetivos comúnmente en contraste entre ellos.

\section{¿Cómo SE ORGANIZAN LOS ACTORES EN TORNO A LA ACCIÓN TERRITORIAL?}

Las iniciativas y acciones que los actores ponen en marcha para construir, gestionar y promover el desarrollo de sus territorios (acción territorial) es un proceso que se construye dentro de un contexto social en donde interactúan, en muchos casos, múltiples actores, en donde cada uno de ellos se moviliza según sus redes de cooperación y solidaridad o sus intereses, pretendiendo influir, bloquear o activar estrategias de acción a partir de los distintos medios que se encuentran a su disposición. Es un campo dinámico, una arena (GIDDENS, 1984), un proceso social tejido de interacciones, en la que concurren diferentes tomas de posición de los múltiples actores involucrados a través de la acción pública, la acción colectiva y la acción privada, con implicancias en el entramado decisional (SUBRA, 2008; FAURE, 2008; MASSON-VINCENT, 2008). A mayor complejidad de las iniciativas y las acciones, mayor complejidad del modelo de organización y gobernanza seguido por los actores para llevar adelante las acciones que construyen territorio.

Las formas de organización de los actores dentro del territorio se han convertido en un elemento clave para dar cuenta como las sociedades construyen su desarrollo. Sin embargo, es importante reconocer que estas formas de organización han cambiado sustancialmente en el último medio siglo, pues de la posición jerárquica que supo cumplir el Estado en los procesos de organización territorial (pues este era el que planificaba, generaba infraestructuras, organizaba los usos del suelo y definía los modelos de desarrollo en general), se pasó a un poder relacional centrado en redes de agentes que intervienen en ámbitos plurisectoriales y en múltiples niveles escalares (regionales, nacionales e internacionales), accionando diversos tejidos y organizaciones sociales que también tienen 
un peso cada vez mayor en la organización de los territorios (MONCAYO JIMÉNEZ, 2001; BOISIER, 2002, 2004; SCHEJTMAN; BERDEGUÉ, 2004). Así, no es solamente la acción pública la que prima en la construcción de acciones territoriales, sino que también concurren otros actores con múltiples lógicas.

Los modelos de organización social involucrados en la acción territorial pueden ser de gestión jerárquica, que centraliza el poder en un agente especifico, a partir del cual surgen los distintos vínculos de interacción (modelo estatal por ejemplo en el cual prima el rol de un gobierno, central, provincial o local, por encima de cualquier otro actor), con una detallada reglamentación; también pueden ser horizontal, que promueve una gestión en redes entre los diferentes actores participantes, que comparten vínculos formales como informales, por ejemplo, una red de organizaciones locales asociadas para promover un proyecto de desarrollo a nivel local y que puede o no estar formalizada en una estructura institucional (agencia de desarrollo local, consorcios de productores, grupos de municipios involucrados en un proyecto de desarrollo regional, etc.), o combinada, que implica una complementariedad entre ambas estructuras, presentándose dinámicas verticales u horizontales en función de la diferentes situaciones que se vayan presentando a lo largo de la acción. La figura 1 da cuenta de estos tres modelos posibles de vinculación entre los actores dentro de la gobernanza local del territorio.

Figura 1: Tipo de vínculo entre actores y la forma de gobernanza

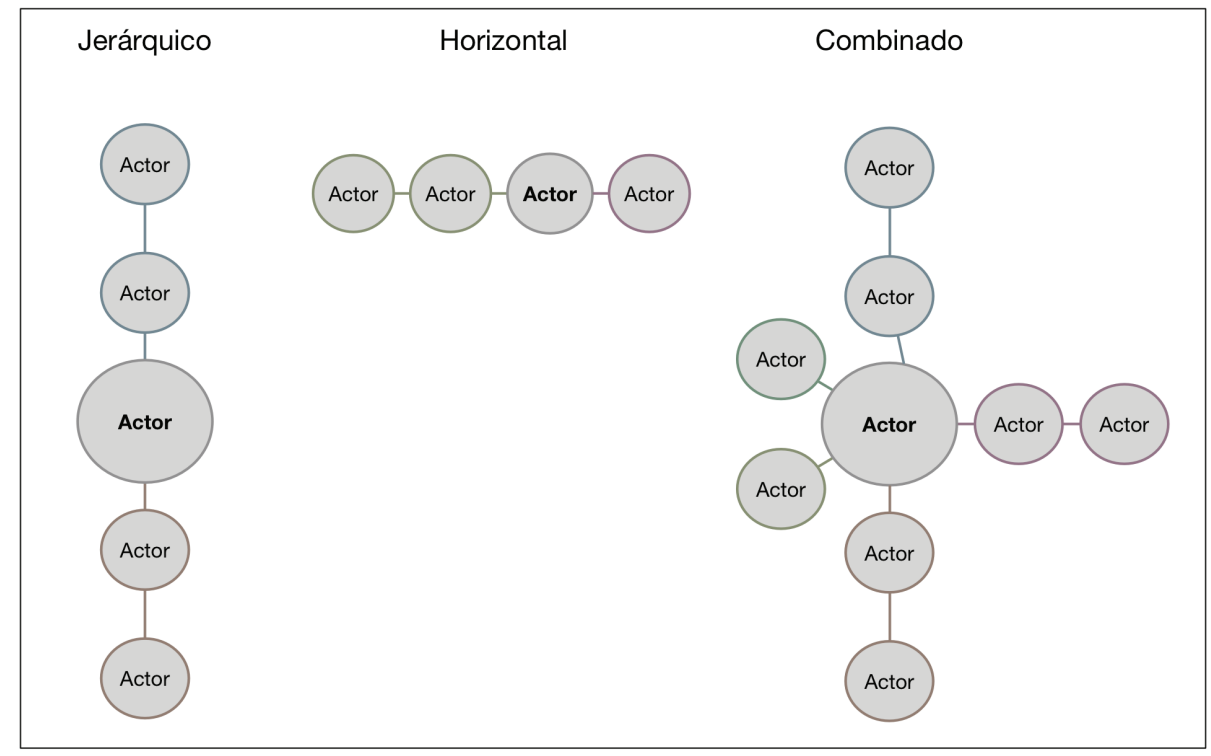

Fuente: Elaboración personal.

Esta interacción entre los actores dentro de esquemas de organización social y de gobernanza se encuentran reguladas por reglas de juego explícitas y generalmente formalizadas jurídicamente e institucionalizadas en estructuras reglamentarias, o bien "pueden ser normas informales, compartidas por los miembros de una organización y que rigen implícitamente los comportamientos en sociedad” (SUBIRATS et al., 2008, p. 98).

Entre las normas formales se destaca, en primer lugar, el marco constitucional, que contiene las reglas constitutivas de un Estado y que se aplica al conjunto de las decisiones, a las libertades individuales o a las autoridades políticas. En segundo lugar, 
las reglas institucionales que regulan las organizaciones administrativas propias del ámbito estatal, refiriendo a aspectos organizacionales y procedimentales, entre ellas, por ejemplo, las reglas específicas referidas del uso y la gestión del espacio (KNOEPFEL et al., 2007).

Por otro lado, están las reglas informales circunscriptas al sistema de valores, símbolos y normas de comportamiento propios de cada sociedad, que proporcionan un marco de significación que guía las acciones. Ciertas convenciones sociales alcanzan tal legitimidad que llegan a sustituir incluso las propias estructuras formales (KNIGHT, 1992).

Este sistema de normas, tanto formales como informales materializa las relaciones de poder entre los grupos sociales, facilitando o limitando las dinámicas de acción. El análisis del juego de actores deberá incluir la dimensión institucional que lo enmarca, identificando las reglas que intervienen, su estabilidad en el tiempo, los procesos de negociación que implican, los posibles conflictos entre ellas y su influencia en la conducta de los participantes.

Es importante reconocer que en todo proceso de construcción territorial, las reglas normativas se van volviendo resistentes al cambio a medida que se consolidan en el tiempo. Sin embargo, estas no son completamente rígidas, al contrario, el actor adecua sus acciones a estas normas, y a su vez lo modifica a partir de sus propias conductas individuales. Las reglas afectan las preferencias de los individuos pero al mismo tiempo éstos las utilizan estratégicamente para hacer valer sus intereses en el escenario deseado de desarrollo (BUSTOS CARA, 2002b; 2008).

\section{LA DINÁMICA DE LA ACCIÓN TERRITORIAL}

La acción territorial es un proceso en el cual múltiples actores organizados a través de formas estatales, colectivas o individuales, van generando iniciativas en el territorio relacionadas con otras acciones y otros actores con los cuales, necesariamente, se abren espacios de intercambio. Esto, a su vez, va generando sinergias que potencian y efectivizan las acciones en marcha o, por el contrario, conflictos y bloqueos que limitan el alcance y la efectividad de las acciones y las iniciativas. Teniendo en cuenta ello, tres elementos son claves e interesa comprender.

En primer lugar, interesa entender la multiescalaridad de la acción territorial, es decir, la capacidad y el tipo de articulación que se construye entre diferentes niveles de organización territorial (en el cual participan los diferentes actores) para poder llevar adelante la acción territorial. Así, existen acciones territoriales que claramente no sólo involucra el territorio en el cual se produce, sino también otros niveles de organización territorial involucrados en estas acciones. La figura 2 da cuenta de esta multiescalaridad. 
Figura 2: Articulación multiescalar de las acciones territoriales en marcha

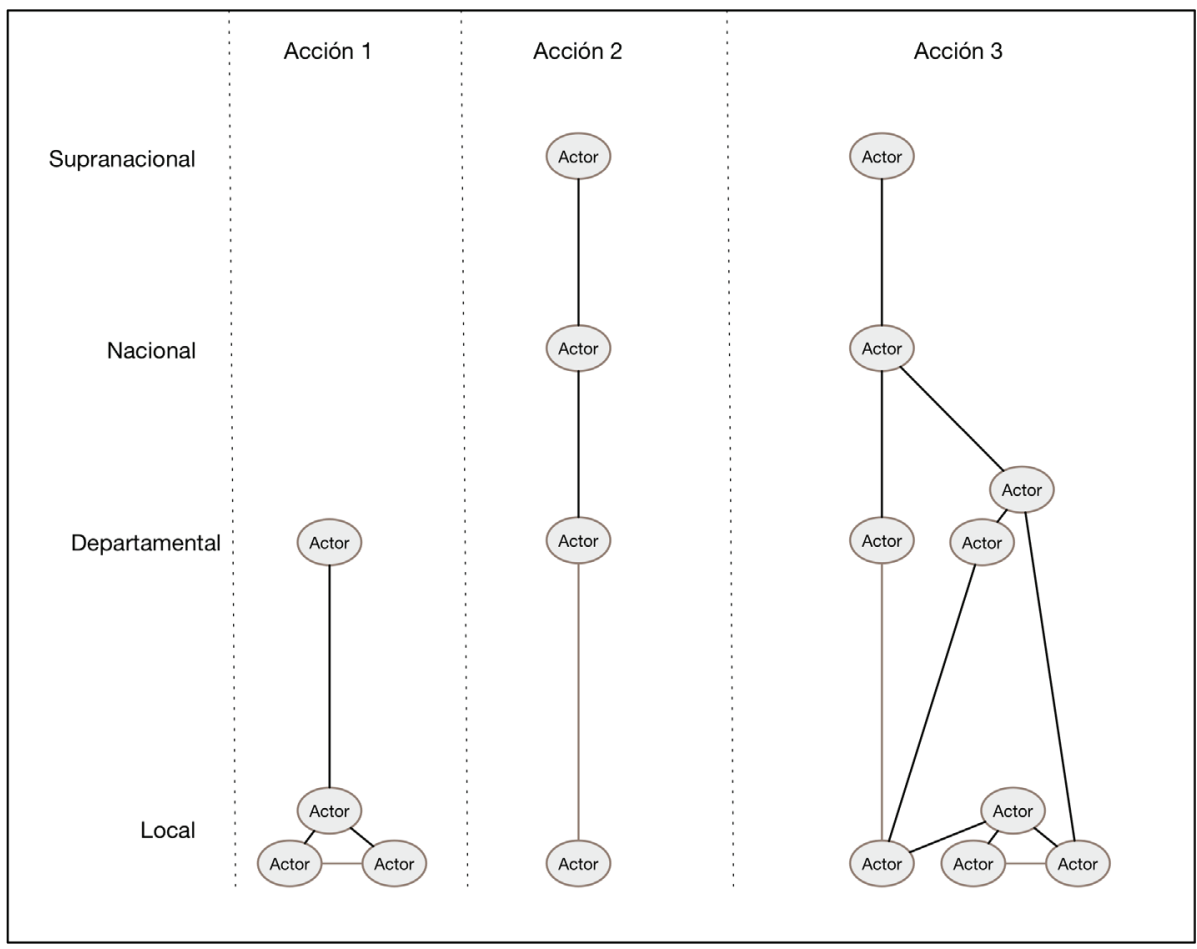

Fuente: Elaboración personal

El segundo factor clave es la construcción de procesos sinergéticos y virtuosos entre las acciones que tienen lugar en un territorio. Así hay acciones que mantienen vinculaciones con otras acciones locales, pues son complementarias o subsidiarias unas con otras, lo que produce un alineamiento de actores y una potenciación de las iniciativas en marcha. Es probable que existan acciones integrales con fuerte densidad de vínculos entre actores y que abarquen problemáticas complejas, por ejemplo, la puesta en marcha de un plan de desarrollo territorial, habrá otros casos en los cuales las acciones serán de carácter más sectorial, con mínimas articulaciones con otras acciones y otros actores tanto de nivel local como de otros niveles escalares. Interesa analizar estos procesos sinérgicos y los encadenamientos que estos generan, pues en definitiva son los que muestran las dinámicas reales de desarrollo de un territorio. La figura 3 pretende dar cuenta de esta coordinación y sinergia entre acciones locales

Finalmente, el tercer elemento clave lo constituye el análisis de los frenos y limitantes a la acción territorial. Interesa observar en cualquier acción territorial cuáles son los frenos o las limitantes que bloquean o anulan las acciones territoriales en marcha. Una clara identificación y tipología de dichos frenos es un elemento clave para poder luego definir estrategias de mejora de las acciones territoriales y del desarrollo en general. 
Figura 3: Articulación y coordinación de las acciones territoriales

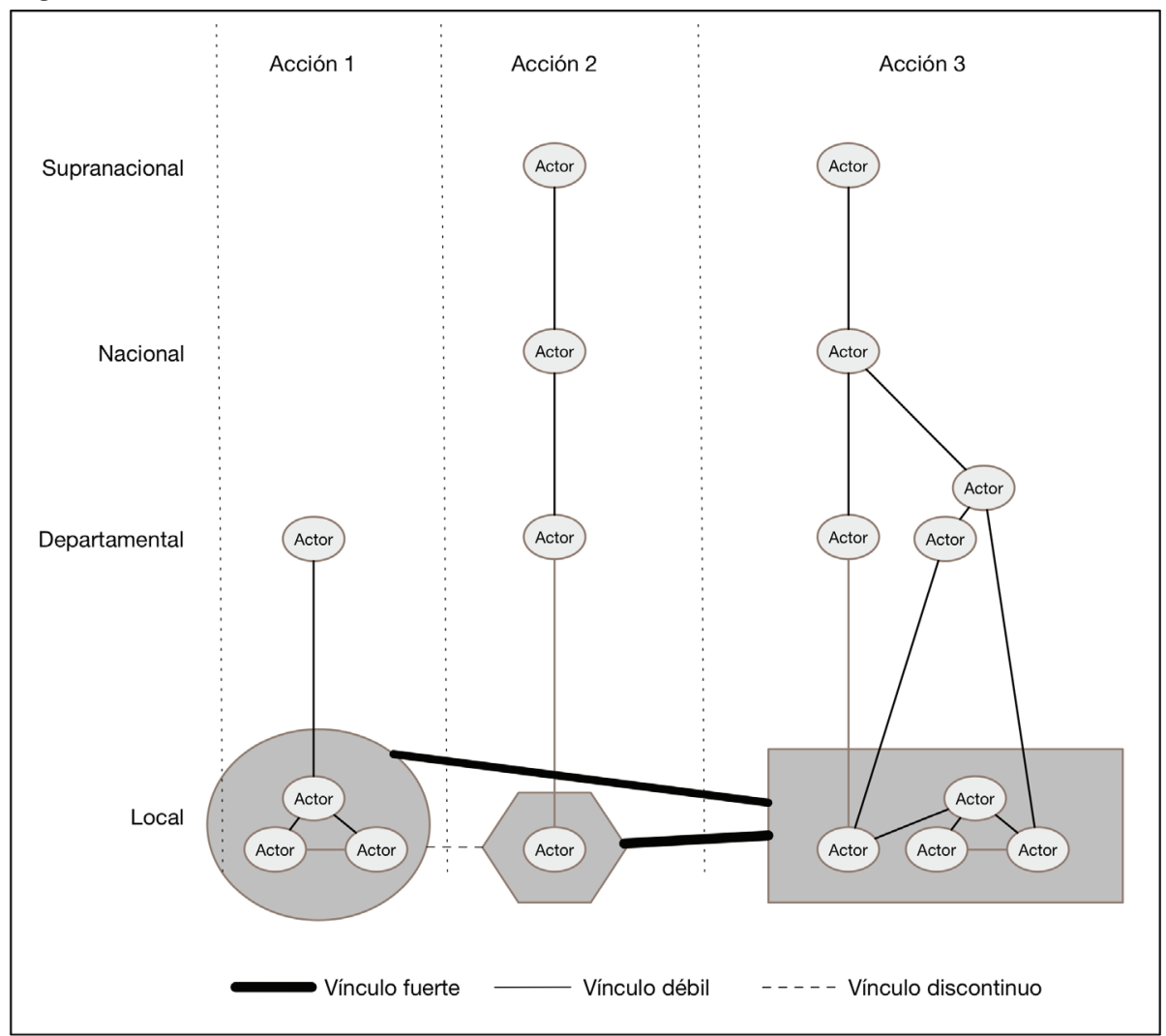

Fuente: Elaboración personal

\section{CONCLUSIÓN}

La aceleración en la transformación de los territorios en las últimas décadas ha incentivado la emergencia de múltiples iniciativas de distinto tenor, orientadas a construir, organizar y mejorar las condiciones de vida y de producción en los mismos, bajo el símbolo inequívoco de un camino hacia el progreso y el desarrollo, visto desde su dimensión occidental y universal. El resultado de esta sobreabundancia de acciones en los territorios no ha dado los resultados esperados: los territorios viven procesos de fragmentación crecientes, con regiones que mejoran ciertas condiciones de vida, con otras regiones que mantienen altos niveles de pobreza y marginalidad.

Las hipótesis acerca del fracaso de las acciones para el desarrollo son múltiples y tienen que ver con la especificidad de cada territorio. Sin embargo, consideramos que antes de pensar en resultados de políticas e iniciativas, es necesario poner el foco en el análisis de cómo se construyen las acciones territoriales y las bases ideológicas y normativas sobre las cuáles se construyen, pues consideramos que allí están las respuestas para entender el desarrollo de los territorios y la sociedad.

El concepto de acción territorial y ciertos elementos metodológicos esbozados aquí, pretenden contribuir al esclarecimiento de las formas como las sociedades construyen su devenir.

Desde el punto de vista epistemológico, es necesario ubicar al análisis de la acción territorial en el contexto de las ciencias de la acción en tanto dicho análisis nos 
Marcelo Sili es geógrafo por la Universidad Nacional del Sur (UNS), Argentina; doctor en Desarrollo Rural por la Université Toulouse Le Mirail, Francia; profesor en el Departamento de Geografía y Turismo de la UNS; investigador del Consejo Nacional de Investigaciones Científicas y Técnicas (CONICET).

E-mail: sili.marcelo@gmail. com

Artículo recibido el 6 de marzo de 2017 y aprobado para publicación el 30 de mayo de 2017. permite entender el juego que se pone en marcha entre la subjetividad y las intencionalidades de los actores (enfoques constructivistas) y las estructuras espaciales locales y los condicionantes globales (enfoques estructuralistas), las cuales en este nuevo contexto histórico, provienen de múltiples lugares, así como de múltiples fuentes de conocimiento.

Por otro lado, en términos de práctica científica, identificar las formas cómo se produce la acción territorial es un elemento de sumo interés, pues permitiría identificar patrones y comportamientos que, una vez modelizados, van a permitir definir estrategias y procedimientos para gestionar el proceso de construcción territorial hacia escenarios de mayor consenso entre los múltiples actores del territorio, los cuales en definitiva serían escenarios con mayores posibilidades de inclusión y sostenibilidad. En otras palabras, aportar un marco interpretativo de la acción territorial que pueda identificar las regularidades entre las coaliciones de actores, asociando a los sujetos a determinadas lógicas de acción y al uso de recursos específicos, sin dudas termina por ofrecer información de gran utilidad para aquellos organismos públicos encargados de definir las estrategias a partir de las cuales gestionar procesos territoriales hacia escenarios deseados.

\section{REFERENCIAS}

ABRAMOVAY, R. Para una teoría de los estudios territoriales. En: MANZANAL, M.; NEIMAN, G.; LATTUADA, M. (Org.). Desarrollo rural: Organizaciones, instituciones y territorios. Buenos Aires: CICCUS, 2006. p. 51-70.

APPADURAI, A. El futuro como hecho cultural. Ensayos sobre la condición global. Buenos Aires: Fondo de Cultura Económica, 2015.

BERDEGUÉ, J. et al. Territorios en Movimiento: Dinámicas Territoriales Rurales en América Latina. Santiago: Centro Latinoamericano para el Desarrollo Rural, 2012. (Documento de Trabajo, 110). Disponible en: <http://otu.opp.gub.uy/sites/default/files/docsBiblioteca/Territorios\%20en\%20Movimiento.pdf>. Acceso en: 27 jul. 2017.

BOISIER, S. 2001: La odisea del desarrollo territorial en américa latina. La búsqueda del desarrollo territorial y de la descentralización. En: SEMINARIO DESCENTRALIZACIÓN DE SECTORES SOCIALES: NUDOS CRÍTICOS Y ALTERNATIVAS, 2002, Lima. Anales... Lima: Ministerios de la Presidencia, de Educación y de Salud del Perú, 2002. Disponible en: $<$ http://www.top.org.ar/ecgp/FullText/000000/BOISIER\%20Sergio\%20 -\%202001\%20La\%20odisea\%20del\%20desarrollo.pdf>. Acceso en: 27 jul. 2017.

. Desarrollo territorial y descentralización. El desarrollo en el lugar y en las manos de la gente. Eure, v. 30, n. 90, p. 27-40, 2004. https://doi.org/10.4067/s025071612004009000003

BOURDIEU, P. Capital cultural, escuela y espacio social. México: Siglo XXI, 2005.

BUSTOS CARA, R. Los sistemas territoriales. Etapas de Estructuración y Desestructuración en Argentina. Anales de Geografía de la Universidad Complutense, v. 22, p. 113-129, 2002a. Disponible en: <http://revistas.ucm.es/index.php/AGUC/article/view/32346>. Acceso en: 27 jul. 2017.

Cambios en los sistemas territoriales. Actores y sujetos entre la estructura y la acción. Propuesta teórico-metodológica. En: JORNADAS INTERDISCIPLINARIAS DEL SUDOESTE BONAERENSE, 2., 2002, Bahía Blanca. Anales... Bahía Blanca: UNS, 2002b. 
Por una geografía de la acción territorial. En: JORNADAS CUYANAS DE GEOGRAFÍA, 10., 2008, Mendoza. Anales... Mendoza: UNCUYO, 2008.

; ALBALADEJO, C. La transformación de las sociedades rurales locales de la provincia de Buenos Aires: el caso de la localidad de Algarrobo, Partido de Villarino. Revista Universitaria de Geografía, v. 6, n. 1, p. 35-62, 1998.

CAMPAGNE, P.; PECQUEUR, B. Le développement territorial. Une réponse émergente à la mondialisation. Paris: Ed. Charles Léopold Mayer, 2014.

CASTELLS, M. La Era de la Información. Economía, Sociedad y Cultura. La Sociedad Red. Madrid: Alianza Editorial, 1999.

COMISIÓN ECONÓMICA PARA AMÉRICA LATINA. The social inequality matrix in Latin America. Santo Domingo: CEPAL, 2016. Disponible en: <http://repositorio.cepal.org/bitstream/handle/11362/40710/S1600945_en.pdf?sequence $=1 \& \mathrm{i}$ sAllowed=y>. Acceso en: 27 jul. 2017.

CROZIER, M.; FRIEDBERG, E. L'acteur et le systeme. Les contraites de l'action colective. París: Seuil, 1977.

DENTE, B.; SUBIRATS, J. Decisiones públicas: Análisis y estudio de los procesos de decisión en políticas públicas. Barcelona: Ariel, 2014.

DICKEN, P. Mudanca Global. Mapeando as Novas Fronteiras da Economia Mundial. 5 ed. Sao Paulo: Bookman, 2010.

FAURE, A. La question territoriale: pouvoirs locaux, action publique et politique(s). Grenoble: Cerat - Institut d'Etudes Politiques de Grenoble - Université Pierre Mendès France, 2008. Disponible en: <https://tel.archives-ouvertes.fr/tel-00326249/document>. Acceso en: 27 jul. 2017.

FRANÇOIS, H.; HIRCZAK, M.; SENIL, N. Territoire et patrimoine: la coconstruction d'une dynamique et de ses ressources. Revue d'Economie Régionale et Urbaine, n. 5, p. 683-700, 2006. https://doi.org/10.3917/reru.065.0683

GIDDENS, A. La Constitución de la Sociedad. Buenos Aires: Amorrortu, 1984.

GUMUCHIAN, H. et al. Les acteurs, ces oubliés du territoire. Paris: Anthropos, 2003.

HARVEY, D. Ciudades rebeldes. Del derecho de la ciudad a la revolución urbana. Buenos Aires: Akal Pensamiento crítico, 2013.

HERNÁNDEZ, O. Fortalecimiento de Alianzas Intersectoriales Solidarias público-no estatales para el desarrollo en ámbitos localescomunitarios. Alncance: Revista Cubana de Información y Comunicación, v. 5, n. 11, p. 69-103, sept. 2016. Disponible en: <http:// www.alcance.uh.cu/index.php/RCIC/article/view/76>. Acceso en: 27 jul. 2017.

JIMÉNEZ, J. M. Planes territoriales y urbanísticos: situación actual y perspectivas de futuro. Review International on Sustainable Housing and Urban Renewal, n. 4, v. 1, 2016.

KEBIR, L. Ressource et développement régional, quels enjeux? Revue d'Économie Régionale \& Urbaine, n. 5, p. 701-723, 2006. https://doi.org/10.3917/reru.065.0701

KNIGHT, H. Institutions and social conflict. Nueva York: Cambridge University Press, 1992.

KNOEPFEL, P. et al. Hacia un modelo de análisis de políticas públicas operativo. Un enfoque basado en los actores, sus recursos y las instituciones. Ciencia Politica, n. 3, p. 6-29, 2007. Disponible en: <https://dialnet.unirioja.es/servlet/articulo?codigo=3663617>. Acceso en: 27 jul. 2017.

LAJARGE, R. Le développement territorial ou comment satisfaire le besoin grandissant de territorialités multiples. Fonder les Sciences Territoriales, p. 128-132, 2011. Disponible en: <https://hal.archives-ouvertes.fr/halshs-00700675/document>. Acceso en: 27 jul. 2017.

LANDEL, P.; PECQUEUR, B. L'operateur territorial, vecteur du changement. En: COLLOQUE ASSOCIATION DE SCIENCE RÉGIONALE DE LANGUE FRANÇAISE, 2011. 
LE MOIGNE, L. La modélisation des systèmes complexes. Aix-en-Provence: Ed. Afcet Systèmes, 1989.

MANZANAL, M.; PONCE, M. (Org.). La desigualdad ¿del desarrollo?. Controversias y disyuntivas del desarrollo rural en el Norte argentino. Buenos Aires: Ed. Ciccus, 2012.

MASSEY, D. Spatial Division of Labour. London: MacMillan, 1985.

Spaces of politics. En: ; ALLEN, J.; SARRE, P. (Ed.). Human Geography Today. Cambridge: Polity Press, 1991. p. 279-294.

MASSON-VINCENT, M. Governance and geography explaining the importance of regional planning to citizens, stakeholders in their living space. Boletin de la A.G.E., n. 46, p. 363-367, 2008.

MOINE, A.; FAIVRE, E. Le territoire comme un système complexe: de la représentation ‘a l'action. En: CONFERENCE INTERCONTINENTALE D'INTELLIGENCE TERRITORIALE "INTERDISCIPLINARITE DANS L'AMENAGEMENT ET DEVELOPPEMENT DES TERRITOIRES". 1., Gatineau, 2011. Annales... International Network of Territorial Intelligence, 2013. Disponible en: <https://hal. archives-ouvertes.fr/halshs-00960901/document>. Acceso en: 27 jul. 2017.

MONCAYO JIMÉNEZ, E. Evolución de los paradigmas y modelos interpretativos del desarrollo territorial. Santiago: Instituto Latinoamericano y del Caribe de Planificación Económica y Social, 2001. (Gestión Pública, 13). Disponible en: <http://repositorio. cepal.org/bitstream/handle/11362/7262/S018637_es.pdf?sequence=1>. Acceso en: 27 jul. 2017.

OHMAE, K. The Borderless World, Power and Strategy in the Interlinked Economy. Londres: Harpercollins, 1990.

PORTER, M. Locations, Clusters, and Company Strategy. En: CLARK, G. L. ; FELDMAN, M. P.; GERTLER, M. S. (Ed.) The Oxford Handbook of Economic Geography. London: Oxford University Press, 2000. p. 253-274.

POUTHIER, F. Pourquoi et comment renouveler l'action territoriale aujourd'hui?. En: PIGNOT, L.; QUILES, J-P. (Coord.). Culture et Territoires: vers de nouvelles coopèrations des acteurs artistiques et culturels. Paris: Librairie des Territoires, Éditions de l'OPC, 2013. p. 28-33.

REAL-DATO, J. Mechanisms of Policy Stability and Change: epistemological and theoretical implications of the application of the Institutional Analysis and Development. En: WORLD CONGRESS OF POLITICAL SCIENCE, 21., Santiago, 2009. Santiago: IPSA, 2009. Disponible en: <https://w3.ual.es/-jreal/papers/Real\%20IPSA\%2009\%20 Mechanisms\%20epistemological\%2020090528.pdf>. Acceso en: 27 jul. 2017.

RIFFO, L. 50 años del ILPES: evolución de los marcos conceptuales sobre desarrollo territorial. Santiago de Chile: CEPAL, 2013 (Desarrollo Territorial, 15). Disponible en: <http://repositorio.cepal.org/bitstream/handle/11362/7248/S1303593_es.pdf?sequence=1\&isAllowed=y>. Acceso en: 27 jul. 2017.

RIST, G. Le développement: histoire d'une croyance occidentale. París: Ed. Sciences Po., 2013.

SASSEN, S. The Global City. New York, London, Tokyo. New Jersey: Princeton University Press, 1991.

SCHEJTMAN, A.; BERDEGUÉ, J. Desarrollo territorial rural. Santiago: Centro Latinoamericano para el Desarrollo Rural, 2004 (Debates y temas rurales, 1). Disponible en: $\quad$ http://www.rimisp.org/wp-content/files_mf/1363093392schejtman_y_ berdegue2004_desarrollo_territorial_rural_5_rimisp_CArdumen.pdf >. Acceso en: 27 jul. 2017.

SILI, M. ¿Cómo revertir la crisis y la fragmentación de los territorios rurales? Ideas y propuestas para emprender procesos de desarrollo territorial rural. Buenos Aires: INTA, 2010.

Un modelo para comprender la dinámica de los territorios rurales. El caso de la 
Argentina. Mundo Agrario, v. 17, n. 34, e003, abr. 2016. Disponible en: <http://www. mundoagrario.unlp.edu.ar/article/view/MAv17n34a03>. Acceso en: 27 jul. 2017.

; BUSTOS CARA, R.; GUIBERT, M. Atlas de la Argentina Rural. Buenos Aires: Capital Intelectual, 2015.

SUBIRATS, J. Politicas públicas e inclusión social. Factores territoriales y gobiernos locales. Barcelona: Instituto de Gobierno y Políticas Públicas, 2010.

et al. Análisis de políticas públicas y gestión pública. Barcelona: Ariel, 2008.

SUBRA, P. L'aménagement, une question géopolitique. Hérodote, v. 130, n. 3, p. 222-250, 2008. https://doi.org/10.3917/her.130.0222

TACOLI, C. Rural-Urban Linkages. London: Earthscan, 2006.

VANIER, M. Demain les territoires. Capitalisme reticulaire et espace politique. Paris: Hermannn, 2015.

VEIGA, J. E. Cidades Imaginárias. Campinas: Autores Associados, 2002.

VELTZ, P. Hiérarchies et réseaux dans l'organisation de la production et du territoire. En: BENKO, G.; LIPIETZ, A. (Dir.). Les régions qui gagnent. Paris: PUF, 1992. p. 293-314. 